Research Article

\title{
Uniqueness in Inverse Electromagnetic Conductive Scattering by Penetrable and Inhomogeneous Obstacles with a Lipschitz Boundary
}

\author{
Fenglong Qu \\ School of Mathematics and Information Science, Yantai University, Yantai, Shandong 264005, China \\ Correspondence should be addressed to Fenglong Qu, fenglongqu@amss.ac.cn
}

Received 26 August 2012; Accepted 6 December 2012

Academic Editor: Yong Hong Wu

Copyright (C) 2012 Fenglong Qu. This is an open access article distributed under the Creative Commons Attribution License, which permits unrestricted use, distribution, and reproduction in any medium, provided the original work is properly cited.

This paper is concerned with the problem of scattering of time-harmonic electromagnetic waves by a penetrable, inhomogeneous, Lipschitz obstacle covered with a thin layer of high conductivity. The well posedness of the direct problem is established by the variational method. The inverse problem is also considered in this paper. Under certain assumptions, a uniqueness result is obtained for determining the shape and location of the obstacle and the corresponding surface parameter $\lambda(x)$ from the knowledge of the near field data, assuming that the incident fields are electric dipoles located on a large sphere with polarization $p \in \mathbb{R}^{3}$. Our results extend those in the paper by F. Hettlich (1996) to the case of inhomogeneous Lipschitz obstacles.

\section{Introduction}

In this paper we are interested in determining the shape and location of a penetrable, inhomogeneous, isotropic, Lipschitz obstacle surrounded by a piecewise homogeneous, isotropic medium. The obstacle is covered with a thin layer of high conductivity. Such penetrable obstacles lead to conductive boundary conditions; for the precise mathematical description, the reader is referred to [1-3]. In this paper, it is shown that the shape and location of the obstacle and the corresponding surface parameter are uniquely determined from a knowledge of the near field data of the scattered electromagnetic wave at a fixed frequency. To this end, we need a well posedness result for the direct problem.

The well posedness of the Helmholtz equation for a penetrable, inhomogeneous, anisotropic medium has been studied recently in [4]. In [5], the authors provided a proof 
for the well posedness of the scattering problem for a dielectric that is partially coated by a highly conductive layer in the TM case in 2007.

In the case of exterior Maxwell problem for the partially coated Lipschitz domains, the authors in [6] have established the well posedness of a unique solution by variational methods in 2004. For the homogeneous isotropic medium problem, by means of an integral equation method, Angell and Kirsch proved the existence and uniqueness of the classical solution for Maxwell's equations with conductive boundary conditions assuming $\lambda \in$ $C^{0, \alpha}(\partial D)$ in [2]. Variational methods for the homogeneous isotropic medium problem were proposed in [1], under the assumption that the bounded domain $D \subseteq \mathbb{R}^{3}$ with boundary $\partial D$ in the class $C^{2}$, and some additional conditions on $k, n_{D}, \mu, \lambda$. It is also shown that the obstacle is uniquely determined by the far field patterns of all incident waves with a fixed wave number. For the inhomogeneous anisotropic media, the well posedness of the direct problem was proved in [7].

The uniqueness result for the inverse medium scattering problem was first provided by Isakov (see $[8,9])$, in which it is shown that the shape of a penetrable, inhomogeneous, isotropic medium is uniquely determined by its far field pattern of all incident plane waves. The idea is to construct singular solutions of the boundary value problem with respect to two different scattering obstacles with identical far field patterns. Our uniqueness proof is based on this idea. The idea of Isakov was modified by Kirsh and Kress [10] using potential theory for the impenetrable obstacle case with Neumann boundary conditions. By the same technique, the authors in [11] proved the case of a penetrable obstacle with constant index of refraction. The use of potential theory will require strong smoothness assumptions on the scattering object. Then D. Mitrea and M. Mitrea [12] improved the previous results to the case of Lipschitz domains. In [13], they extended Isakov's approach to the case of a penetrable obstacle for Hemholtz equations. The uniqueness theorem of Helmholtz equations for partially coated buried obstacle problem was shown in [14, 15], assuming that the scattering fields were known with point sources as incident fields.

Recently, uniqueness for the inverse scattering problem in a layered medium has attracted intensive studies. For the sound-soft or sound-hard obstacle case, based on Schiffer's idea, [16] proved a uniqueness result. But their method can not be extended to other boundary conditions. In recent years, by employing the generalized mixed reciprocity relation, it was proved in $[17,18]$ that both the obstacle and its physical property can be uniquely determined for different boundary conditions. For the inverse acoustic scattering by an impenetrable obstacle in a two-layered medium case, it is shown in [19] that interface is uniquely determined from the far field pattern. Unfortunately, this method can not be extended to the electromagnetic case, but using ideas in [20], a different method was used in [21] to establish such a uniqueness result for the electromagnetic case.

There are also some uniqueness results for partial differential equations with constant coefficients by integral equation methods. (see [22, 23]). However, integral equation methods are not well tailored for partial differential equations having inhomogeneous coefficients of the highest derivatives. Consequently, in [24], the author brought together the variational approach and the idea from $[8,9]$ to provide a uniqueness proof of Helmholtz equations with inhomogeneous coefficients for a penetrable, anisotropic obstacle. Their method depends on a regularity theorem for the direct problem and the well posedness of the interior transmission problem related to the direct problem. This idea has been extended to the case of electromagnetic scattering problem for anisotropic media in [25].

The outline of this paper is as follows. In Section 2, besides the formulation of the direct scattering problem in a penetrable, inhomogeneous, Lipschitz domain, we also provide 
a proof of the well posedness for the direct problem by using a variational method. The uniqueness result for the inverse problem will be shown in Section 3.

\section{The Direct Problem}

Let $D \subset \mathbb{R}^{3}$ be a bounded penetrable, inhomogeneous, isotropic domain with a Lipschitz boundary $\partial D$ denoted by $\Gamma$ and covered with a thin layer of high conductivity. Assume that the domain $D$ is imbedded in a homogeneous background medium. Define $k_{D}^{2}=k^{2} n_{D}(x)$ and $k_{b}^{2}=k^{2} n_{b}$ with $k>0$ being the wave number, where $n_{D}(x)$ and $n_{b}$ are the refractive index of the domain $D$ and the background medium, respectively. Assume that $n_{D} \in C(\bar{D})$ with $\operatorname{Re}\left[n_{D}(x)\right]>0, \operatorname{Im}\left[n_{D}(x)\right]>0$ for all $x \in D$ and $n_{b}$ is a complex constant with $\operatorname{Im}\left(n_{b}\right) \geq 0$. Assume further that $\lambda \in L^{\infty}(\Gamma)$ with $\operatorname{Re}[\lambda(x)] \geq 0$ is a complex-valued function describing the surface impedance of the coating. The incident field is considered to be an electric dipole located at $x_{0}$ on a large sphere $S_{R_{0}}=\left\{x \in \mathbb{R}^{3}:|x|=R_{0}\right\}$ with polarization $p \in \mathbb{R}^{3}$ given by

$$
E_{e}\left(x, x_{0}, p, k_{b}\right)=\frac{i}{k_{b}} \operatorname{curl}_{x} \operatorname{curl}_{x} p \frac{e^{i k_{b}\left|x-x_{0}\right|}}{4 \pi\left|x-x_{0}\right|}
$$

Denote by $G\left(x, x_{0}\right)$ the free space Green tensor of the background medium and define $E^{i}(x)=$ $E^{i}\left(x, x_{0}, p\right)=p G\left(x, x_{0}\right)$ which satisfies

$$
\operatorname{curlcurl} E^{i}(x)-k_{b}^{2} E^{i}(x)=p \delta\left(x-x_{0}\right) \text { in } \mathbb{R}^{3},
$$

where $\delta$ is the Dirac delta function. Note that $E^{i}(x)$ can be written as

$$
E^{i}(x)=E_{e}\left(x, x_{0}, p, k_{b}\right)+E_{b}^{s}(x)
$$

where $E_{b}^{S}(x)$ is the scattered electric field due to the background medium and the electric dipole $E_{e}\left(x, x_{0}, p, k_{b}\right)$.

In order to formulate precisely the scattering problem, recall the following Sobolev spaces:

$$
\begin{gathered}
H(\operatorname{curl}, D)=\left\{u \in\left(L^{2}(D)\right)^{3}, \operatorname{curl} u \in\left(L^{2}(D)\right)^{3}\right\} \\
H(\operatorname{div}, D)=\left\{u \in\left(L^{2}(D)\right)^{3}, \operatorname{div} u \in\left(L^{2}(D)\right)^{3}\right\} \\
L_{t}^{2}(\partial D)=\left\{u \in\left(L^{2}(\partial D)\right)^{3}, v \cdot u=0 \text { on } \partial D\right\},
\end{gathered}
$$




$$
\begin{aligned}
H_{\operatorname{div}}^{-1 / 2}(\partial D) & =\left\{u \in\left(H^{-1 / 2}(\partial D)\right)^{3}, v \cdot u=0, \operatorname{div}_{\partial D} u \in H^{-1 / 2}(\partial D)\right\}, \\
H_{\text {curl }}^{-1 / 2}(\partial D) & =\left\{u \in\left(H^{-1 / 2}(\partial D)\right)^{3}, v \cdot u=0, \operatorname{curl}_{\partial D} u \in H^{-1 / 2}(\partial D)\right\}, \\
H_{0}(\operatorname{curl}, D) & =\left\{u \in\left(L^{2}(D)\right)^{3}, \operatorname{curl} u \in\left(L^{2}(D)\right)^{3}, v \times u=0 \text { on } \partial D\right\},
\end{aligned}
$$

where $v$ denotes the exterior unit normal to $\partial D$. If $D$ is unbounded, we denote by $H_{\text {loc }}($ curl, $D)$ the space of functions $u \in H($ curl, $K)$ for any compact set $K \subset \subset D$. Introduce the space

$$
\tilde{X}=\left\{u \in H_{\text {loc }}\left(\operatorname{curl}, \mathbb{R}^{3}\right),\left.u_{T}\right|_{\Gamma} \in L_{t}^{2}(\Gamma)\right\}
$$

where $u_{T}=v \times u \times v$. Then the scattering problem can be formulated as follows. Given $E^{i}$, find the field $V$ and the scattered field $E^{s}$ such that

$$
\begin{gathered}
\text { curlcurl } V-k_{D}^{2} V=0, \quad \text { in } D, \\
\operatorname{curlcurl} E^{s}-k_{b}^{2} E^{s}=0, \quad \text { in } D_{e}, \\
v \times E^{s}-v \times V=-v \times E^{i}, \quad \text { on } \Gamma, \\
v \times \operatorname{curl} E^{s}-v \times \operatorname{curl} V=i k \lambda E_{T}^{s}+i k \lambda E_{T}^{i}-v \times \operatorname{curl} E^{i}, \quad \text { on } \Gamma,
\end{gathered}
$$

and the scattered field $E^{s}=E-E^{i}$ is required to satisfy the Silver-Müller radiation condition

$$
\lim _{r \rightarrow \infty} r\left(\operatorname{curl} E^{s} \times \hat{x}-i k E^{s}\right)=0
$$

uniformly in $\widehat{x}=x /|x|$, where $r=|x|$.

We first have the following uniqueness result for the above scattering problem.

Theorem 2.1. The scattering problem (2.6)-(2.9) has at most one solution.

Proof. To prove the theorem, it is enough to consider the case $E^{i}=0$ whence $E=E^{s}$. Taking the dot product of (2.6) with $V$ over $D$ and of (2.7) with $E$ over $D_{R}=D_{e} \cap B_{R}$ with $D_{e}=\mathbb{R}^{3} \backslash \bar{D}$, respectively, and integrating by parts, we obtain by using the conductive conditions (2.8) and (2.9) that

$$
\begin{gathered}
\int_{D}\left(|\operatorname{curl} V|^{2}-k_{D}^{2}|V|^{2}\right) d x+\int_{D_{R}}\left(|\operatorname{curl} E|^{2}-k_{b}^{2}|E|^{2}\right) d x \\
-i k \int_{\Gamma} \lambda\left|E_{T}\right|^{2} d s+i k \int_{S_{R}} v \times H \cdot \bar{E}_{T} d s=0,
\end{gathered}
$$


where $H=(1 / i k) \operatorname{curl} E$ is the corresponding scattered magnetic field. Taking the complex conjugate of both sides of (2.11) and using the fact that $\operatorname{Im}\left(n_{D}\right), \operatorname{Im}\left(n_{b}\right)$, and $\operatorname{Re}(\lambda)$ are nonnegative gives

$$
\begin{aligned}
\operatorname{Re} \int_{S_{R}} v \times E \cdot \bar{H} d s= & -k^{-1} \int_{D} \operatorname{Im}\left(n_{D}\right)|V|^{2} d x-k^{-1} \int_{D_{R}} \operatorname{Im}\left(n_{b}\right)|E|^{2} d x \\
& -\int_{\Gamma} \operatorname{Re}(\lambda)\left|E_{T}\right|^{2} d s \leq 0 .
\end{aligned}
$$

An application of the Rellich lemma yields that $E=0$ in $\mathbb{R}^{3} \backslash \bar{B}_{R}$ (see [26, Theorem 6.10]). This, together with the unique continuation principle, implies that $E=0$ in $\mathbb{R}^{3} \backslash \bar{D}$. From the trace theorem, it follows that $v \times E=0$ on $\Gamma$. Thus, taking the imaginary part of (2.11) and using the assumption that $\operatorname{Im}\left[n_{D}(x)\right]>0$ for all $x \in D$, we have that $V=0$ in $D$.

Introduce the electric-to-magnetic Calderon operator $G_{e}$ (see [27]), which maps the electric field boundary data $\varphi$ on the surface of a large ball $B_{R}=\left\{x \in \mathbb{R}^{3}:|x|<R\right\}$ to the magnetic boundary data $\widehat{x} \times H$ on $S_{R}=\partial B_{R}$, where $(E, H)$ satisfies

$$
\begin{gathered}
\operatorname{curl} E-i k H=0, \quad \text { in } \mathbb{R}^{3} \backslash \bar{B}_{R}, \\
\operatorname{curl} H+i k E=0, \quad \text { in } \mathbb{R}^{3} \backslash \bar{B}_{R}, \\
\widehat{x} \times E=\varphi, \quad \text { on } S_{R}, \\
\lim _{r \rightarrow \infty}(H \times x-r E)=0 .
\end{gathered}
$$

Then the scattering problem (2.6)-(2.10) can be reformulated in the following mixed conductive boundary value problem (MOCKUP) over a bounded domain:

$$
\begin{gathered}
\text { curlcurl } V-k_{D}^{2} V=0, \quad \text { in } D, \\
\operatorname{curlcurl} E^{s}-k_{b}^{2} E^{s}=0, \quad \text { in } D_{R}, \\
v \times E^{s}-v \times V=-v \times E^{i}, \quad \text { on } \Gamma, \\
v \times \operatorname{curl} E^{s}-v \times \operatorname{curl} V=i k \lambda E_{T}^{s}+i k \lambda E_{T}^{i}-v \times \operatorname{curl} E^{i}, \quad \text { on } \Gamma, \\
v \times \frac{1}{i k} \operatorname{curl} E^{s}=G_{e}\left(v \times E^{s}\right), \quad \text { on } S_{R},
\end{gathered}
$$

where $D_{R}=D_{e} \cap B_{R}$.

In the following, we introduce some properties of the Calderon operator that will be frequently used in the rest of this section. The basis functions for tangential fields on a sphere $S_{R}$ are the vector spherical harmonics of order $n$ given by

$$
U_{n}^{m}=\frac{1}{\sqrt{n(n+1)}} \nabla_{\tau_{1}} Y_{n}^{m}, \quad V_{n}^{m}=\widehat{x} \times U_{n}^{m}
$$


for $n=1,2, \ldots$ and $m=-n, \ldots, n$. Here, as usual, $\nabla_{\tau_{1}}$ denotes the surface gradient on the surface of the unit sphere $S_{1}$. defined by

For $\varphi \in H_{\mathrm{div}}^{-1 / 2}\left(S_{R}\right)$ given by $\varphi=\sum_{n=1}^{\infty} \sum_{m=-n}^{n} a_{n, m} U_{n}^{m}+b_{n, m} V_{n}^{m}$, the operator $G e$ can be

$$
\mathrm{Ge} \varphi=\sum_{n=1}^{\infty} \sum_{m=-n}^{n}\left\{-i k R \frac{b_{n, m}}{\delta_{n}} U_{n}^{m}+\frac{a_{n, m} \delta_{n}}{i k R} V_{n}^{m}\right\}
$$

where

$$
\delta_{n}=k R \frac{\left(h_{n}^{(1)}\right)^{\prime}(k R)}{h_{n}^{(1)}(k R)}+1
$$

and $h_{n}^{(1)}(k R)$ is the spherical Hankel function.

If $k=i$ in (2.23), we will obtain another operator $\widetilde{G_{e}}$. Properties of $G e$ and $\widetilde{G_{e}}$ are collected in the following lemma (for a proof see [27]).

Lemma 2.2. The operator $\widetilde{G}_{e}$ is negative definite in the sense that

$$
\left\langle\widetilde{G_{e}} \varphi, \varphi \times \widehat{x}\right\rangle_{S_{R}}<0
$$

for any $\varphi \in H_{\mathrm{div}}^{-1 / 2}\left(S_{R}\right)$ with $\varphi \neq 0$. Furthermore,

$$
\begin{gathered}
\left|\left\langle\widetilde{G_{e}} \varphi, \varphi \times \widehat{x}\right\rangle_{S_{R}}\right| \geq C\|\varphi\|_{H_{\operatorname{div}}^{-1 / 2}\left(S_{R}\right)^{\prime}}^{2} \quad \forall \varphi \in H_{\operatorname{div}}^{-1 / 2}\left(S_{R}\right), \\
G e+i k \widetilde{G}_{e}: H_{\operatorname{div}}^{-1 / 2}\left(\operatorname{div}, S_{R}\right) \longrightarrow H_{\operatorname{div}}^{-1 / 2}\left(S_{R}\right)
\end{gathered}
$$

is compact, where

$$
H_{\operatorname{div}}^{-1 / 2}\left(\operatorname{div}, S_{R}\right)=\left\{\varphi=\left.\sum_{n=1}^{\infty} \sum_{m=-n}^{n} b_{n, m} V_{n}^{m}\left|\sum_{n=1}^{\infty} \sum_{m=-n}^{n} \frac{1}{\sqrt{1+n(n+1)}}\right| b_{n, m}\right|^{2}<\infty\right\}
$$

In the remainder of this paper we will refer to (2.17)-(2.21) as (CBP). Here we will adapt the variational approach used in $[6,27]$ to prove the existence of a unique solution to our (CBP). Define

$$
X=\left\{u \in H_{\mathrm{loc}}\left(\operatorname{curl}, B_{R}\right),\left.\quad u_{T}\right|_{\Gamma} \in L_{t}^{2}(\Gamma)\right\}
$$


where $D \subset B_{R}$. Then multiplying (2.17) and (2.18) by test function $\phi \in H_{\text {loc }}$ (curl, $\left.B_{R}\right)$, using formally integration by parts and using the conductive boundary conditions on $\Gamma$, we can derive the following equivalent variational formulation for (CBP). Find $w \in X$ such that

$$
\begin{aligned}
& \int_{D}\left(\operatorname{curl} w \cdot \operatorname{curl} \bar{\phi}-k_{D}^{2} w \cdot \bar{\phi}\right) d x+\int_{D_{R}}\left(\operatorname{curl} w \cdot \operatorname{curl} \bar{\phi}-k_{b}^{2} w \cdot \bar{\phi}\right) d x-i k \int_{\Gamma} \lambda w_{T} \cdot \bar{\phi}_{T} d s \\
& \quad+i k \int_{S_{R}} G_{e}(v \times w) \cdot \bar{\phi}_{T} d s=i k \int_{S_{R}}\left[G_{e}\left(v \times E^{i}\right)-v \times H^{i}\right] \cdot \bar{\phi}_{T} d s
\end{aligned}
$$

where $H^{i}=\left(1 / i k_{b}\right)$ curl $E^{i}$ is the incident magnetic field and

$$
w= \begin{cases}V, & x \in D, \\ E=E^{i}+E^{s}, & x \in \mathbb{R}^{3} \backslash \bar{D} .\end{cases}
$$

We rewrite (2.29) as the problem of finding $w \in X$ such that

$$
A(w, \phi)=B(\phi)
$$

where the sesquilinear form $A: X \times X \rightarrow C$ is defined by

$$
\begin{gathered}
A(w, \phi)=(\operatorname{curl} w, \operatorname{curl} \phi)_{D}-\left(k_{D}^{2} w, \phi\right)_{D}+(\operatorname{curl} w, \operatorname{curl} \phi)_{D_{R}}-\left(k_{b}^{2} w, \phi\right)_{D_{R}} \\
-i k\left\langle\lambda w_{T}, \phi_{T}\right\rangle_{\Gamma}+i k\left\langle G_{e}(v \times w), \phi_{T}\right\rangle_{S_{R^{\prime}}} \\
B(\phi)=i k\left\langle G_{e}\left(v \times E^{i}\right)-v \times H^{i}, \phi_{T}\right\rangle_{S_{R}} .
\end{gathered}
$$

Here $(\cdot, \cdot)_{D}$ denotes the $\left(L^{2}(D)\right)^{3}$ scalar product, and $\langle\cdot, \cdot\rangle_{\partial D}$ denotes the $\left(L^{2}(\partial D)\right)^{3}$ scalar product. We will use a Helmholtz decomposition to factor out the nullspace of the curl operator and then to prove the existence of a unique solution to (CBP).

Define

$$
S=\left\{p \in H^{1}(D) \cap H^{1}\left(D_{R}\right) \mid p=\text { on } \Gamma\right\}
$$

then we seek $p \in S$ such that

$$
A(\nabla p, \nabla \xi)=B(\nabla \xi), \quad \forall \xi \in S
$$

The variational problem (2.34) can be rewritten as

$$
A_{1}(p, \xi)+A_{2}(p, \xi)=B(\nabla \xi), \quad \forall \xi \in S
$$


where we define

$$
\begin{gathered}
A_{1}(p, \xi)=-\left(k_{D}^{2} \nabla p, \nabla \xi\right)_{D}-\left(k_{b}^{2} \nabla p, \nabla \xi\right)_{D_{R}}+k^{2}\left\langle\widetilde{G_{e}}(v \times \nabla p), \nabla_{\tau} \xi\right\rangle_{S_{R}}{ }^{\prime} \\
A_{2}(p, \xi)=i k\left\langle G e+i k \widetilde{G_{e}}(v \times \nabla p), \nabla_{\tau} \xi\right\rangle_{S_{R}} .
\end{gathered}
$$

Here we have used $\nabla_{\tau} \xi$ to write the tangential component of the gradient of $\xi$ in terms of the tangential gradient on the sphere $S_{R}$. By Lemma 2.2, it follows that $\widetilde{\mathrm{G}_{e}}$ is negative definite, then we obtain that $A_{1}(p, \xi)$ is a coercive sesquilinear form on $S \times S$. Further by Lax-Milgram theorem, it is easy to see that $A_{1}(p, \xi)$ gives rise to a bijective operator. Since $v \times \nabla p \in H_{\mathrm{div}}^{-1 / 2}\left(\operatorname{div}, S_{R}\right)$, still by Lemma 2.2 , we know that $A_{2}(p, \xi)$ gives rise to a compact operator. In order to apply the Fredholm alternative to the variational problem (2.34), we need to prove the following uniqueness lemma.

Lemma 2.3. The variational problem (2.34) has at most one solution.

Proof. It suffices to consider the following equation:

$$
-\left(k_{D}^{2} \nabla p, \nabla \xi\right)_{D}-\left(k_{b}^{2} \nabla p, \nabla \xi\right)_{D_{R}}+i k\left\langle G_{e}(v \times \nabla p), \nabla_{\tau} \xi\right\rangle_{S_{R}}=0, \quad \forall \xi \in S
$$

Choosing $\xi=p$, it is easy to see that

$$
i k\left\langle G_{e}(v \times \nabla p), \nabla_{\tau} p\right\rangle_{S_{R}}=\left(k_{D}^{2} \nabla p, \nabla p\right)_{D}+\left(k_{b}^{2} \nabla p, \nabla p\right)_{D_{R}}
$$

By the definition of the operator $G_{e}$, if $E^{s} \in H_{\text {loc }}\left(\operatorname{curl}, \mathbb{R}^{3} \backslash \bar{B}\right)$ is the weak solution of the problem

$$
\begin{gathered}
\text { curlcurl } E^{s}-k^{2} E^{s}=0, \quad \text { in } \mathbb{R}^{3} \backslash \bar{B}, \\
v \times E^{S}=v \times \nabla p, \quad \text { on } S_{R}, \\
\lim _{r \rightarrow \infty}\left(\operatorname{curl} E^{S} \times x-i k r E^{S}\right)=0,
\end{gathered}
$$

then we have

$$
G_{e}(v \times \nabla p)=v \times H^{s}, \quad \text { on } S_{R}
$$

where

$$
H^{S}=\frac{1}{i k} \operatorname{curl} E^{S}
$$


Furthermore, we can compute that

$$
\begin{aligned}
\int_{S_{R}} H^{s} \cdot \overline{v \times E^{s}} d s & =-\left\langle v \times H^{s}, \nabla_{\tau} p\right\rangle_{S_{R}}=-\left\langle G e(v \times \nabla p), \nabla_{\tau} p\right\rangle_{S_{R}} \\
& =-\frac{1}{i k}\left(k_{D}^{2} \nabla p, \nabla p\right)_{D}-\frac{1}{i k}\left(k_{b}^{2} \nabla p, \nabla p\right)_{D_{R}{ }^{\prime}}
\end{aligned}
$$

which together with the fact $\operatorname{Im} n_{D} \geq 0, \operatorname{Im} n_{b} \geq 0$ implies

$$
\begin{aligned}
\operatorname{Re} \int_{S_{R}} v \times E^{s} \cdot \overline{H^{s}} d s & =\operatorname{Re} \int_{S_{R}} H^{s} \cdot \overline{v \times E^{s}} d s \\
& =-\frac{1}{k}\left(\operatorname{Im} k_{D}^{2} \nabla p, \nabla p\right)_{D}-\frac{1}{k}\left(\operatorname{Im} k_{b}^{2} \nabla p, \nabla p\right)_{D_{R}} \leq 0 .
\end{aligned}
$$

Therefore the Rellich lemma ensures us that $E^{s}=0$ in $\mathbb{R}^{3} \backslash \bar{B}$. From (2.39), we see that $\nabla_{\tau} p=0$ on $S_{R}$ and then $\left(k_{D}^{2} \nabla p, \nabla p\right)_{D}+\left(k_{b}^{2} \nabla p, \nabla p\right)_{D_{R}}=0$ which, together with the fact that $\left.p\right|_{\Gamma}=0$, implies $p=0$. This completes the proof of Lemma 2.3.

Lemma 2.3 together with the Fredholm alternative implies that there exits a unique solution $p_{0} \in S$ of the variational problem (2.34).

Lemma 2.4. The space

$$
X_{0}=\{u \in X \mid A(u, \nabla \xi)=0, \forall \xi \in S\}
$$

is compactly imbedded in $\left(L^{2}(B)\right)^{3}$, where $B$ is a ball with $D \subset B \subset B_{R}$.

Proof. Consider a bounded set of functions $\left\{u_{j}\right\}_{j=1}^{\infty} \subset X_{0}$. Each function $u_{j} \in X_{0}$ can be extended to all of $\mathbb{R}^{3}$ by solving the exterior Maxwell equation

$$
\begin{gathered}
\nabla \times\left(\nabla \times v_{j}\right)-k_{b}^{2} v_{j}=0, \quad \text { in } \mathbb{R}^{3} \backslash \bar{B}, \\
v \times v_{j}=v \times u_{j}, \quad \text { on } S_{R}, \\
\lim _{|x| \rightarrow \infty}|x|\left(\left(\nabla \times v_{j}\right) \times v-i k v_{j}\right)=0 .
\end{gathered}
$$

Define

$$
u_{j}^{e}= \begin{cases}u_{j}, & \text { if } x \in B \\ v_{j}, & \text { if } x \in \mathbb{R}^{3} \backslash \bar{B}\end{cases}
$$


Since the tangential components of $u_{j}^{e}$ are continuous across $S_{R}$, it follows that $u_{j}^{e} \in$ $H_{\text {loc }}\left(\right.$ curl, $\left.\mathbb{R}^{3}\right)$. By using the properties of the Calderon operator $G_{e}$ and the conditions in $X_{0}$, we see that the following equations hold true

$$
\begin{gathered}
\operatorname{div}\left(k_{D}^{2} u\right)=0, \quad \text { in } D, \\
\operatorname{div}\left(k_{b}^{2} u\right)=0, \quad \text { in } D_{R} \\
k_{b}^{2} v \cdot u=-i k \nabla_{\tau} \cdot G_{e}(v \times u), \quad \text { on } S_{R} .
\end{gathered}
$$

Then, by the definition of $G_{e}$ that $G e\left(v \times u_{j}\right)=(1 / i k) \mathcal{v} \times \operatorname{curl} v_{j}$ and the relationship $\nabla_{\tau} \cdot(v \times$ $\left.v_{j}\right)=-v \cdot \operatorname{curl} v_{j}$ on $S_{R}$, we immediately have

$$
\begin{aligned}
k_{b}^{2} v \cdot u_{j} & =-i k \nabla_{\tau} \cdot G_{e}\left(v \times u_{j}\right)=-\nabla_{\tau} \cdot\left(v \times \operatorname{curl} v_{j}\right)=v \cdot \operatorname{curl} \text { curl } v_{j} \\
& =k_{b}^{2} v \cdot v_{j}, \text { on } S_{R} .
\end{aligned}
$$

Thus, $u_{j}^{e}$ has a well-defined divergence and $\nabla \cdot\left(\widehat{k}^{2} u_{j}^{e}\right)=0$ in $\mathbb{R}^{3} \backslash \Gamma$, where

$$
\widehat{k}^{2}= \begin{cases}k_{D^{\prime}}^{2} & x \in D \\ k_{b}^{2} & x \in \mathbb{R}^{3} \backslash \bar{D} .\end{cases}
$$

Now we choose a cut-off function $\chi \in C_{0}^{\infty}\left(\mathbb{R}^{3}\right)$ such that $\chi=1$ in $\bar{B}$ and $\chi$ is supported in a ball $B_{R_{1}} \supset \bar{B}$. Then one can use the general compactness theorem (Theorem 4.7 in [27]) to the sequence $\left\{\chi u_{j}^{e}\right\}$ and extract a subsequence converging strongly in $\left(L^{2}(B)\right)^{3}$. This proves the lemma.

From the above definitions of $S$ and $X_{0}$, we have the following Helmholtz decomposition lemma.

Lemma 2.5. The spaces $\nabla S$ and $X_{0}$ are closed subspaces of $X$. The space $X$ is the direct sum of the spaces $\nabla S$ and $X_{0}$, that is,

$$
X=X_{0} \oplus \nabla S .
$$

The proof of this Helmholtz decomposition Lemma is entirely classical (see $[27,28])$.

We now look for a solution of the variational problem (2.31) in the form $w=w_{0}+\nabla p_{0}$, where $w_{0} \in X_{0}$ and $p_{0} \in S$ is the unique solution of (2.34). We observe that $A\left(w_{0}, \nabla \xi\right)=0$ for all $\xi \in S$ by the definition of $X_{0}$. Hence the problem of determining $w \in X$ is equivalent to the problem of determining $w_{0} \in X_{0}$ such that

$$
A\left(w_{0}, \phi_{0}\right)=B\left(\phi_{0}\right)-A\left(\nabla p_{0}, \phi_{0}\right), \quad \forall \phi_{0} \in X_{0}
$$


From Chapter 10.3.2 in [27] we know that for $\varphi \in H_{\operatorname{div}}^{-1 / 2}\left(S_{R}\right)$

$$
G_{e} \varphi=G_{1 e} \varphi+G_{2 e} \varphi
$$

where the operator $G_{1 e}$ is a compact operator from $X_{0}$ into $H_{\text {div }}^{-1 / 2}\left(S_{R}\right)$ and the operator $G_{2 e}$ satisfies $i k\left\langle G_{2 e}(v \times \varphi), \varphi_{T}\right\rangle_{S_{R}} \geq 0$. We now split the sesquilinear form $A(\cdot, \cdot)$ into $A=a+b$ with

$$
\begin{gathered}
a\left(w_{0}, \phi_{0}\right)=\left(\operatorname{curl} w_{0}, \operatorname{curl} \phi_{0}\right)_{D}+\left(w_{0}, \phi_{0}\right)_{D}+\left(\operatorname{curl} w_{0}, \operatorname{curl} \phi_{0}\right)_{D_{R}}+\left(w_{0}, \phi_{0}\right)_{D_{R}} \\
-i k\left\langle\lambda\left(w_{0}\right)_{T},\left(\phi_{0}\right)_{T}\right\rangle_{\Gamma}+i k\left\langle G_{2 e}\left(v \times w_{0}\right),\left(\phi_{0}\right)_{T}\right\rangle_{S_{R^{\prime}}} \\
b\left(w_{0}, \phi_{0}\right)=-\left(\left(k_{D}^{2}+1\right) w_{0}, \phi_{0}\right)_{D}-\left(\left(k_{b}^{2}+1\right) w_{0}, \phi_{0}\right)_{D_{R}}+i k\left\langle G_{1 e}\left(v \times w_{0}\right),\left(\phi_{0}\right)_{T}\right\rangle_{S_{R}} .
\end{gathered}
$$

The sesquilinear form $a(\cdot, \cdot)$ is obviously bounded and a direct computation verifies that

$$
\left|a\left(w_{0}, w_{0}\right)\right| \geqslant \alpha\left\|w_{0}\right\|_{X}^{2}, \quad \forall w_{0} \in X_{0}
$$

with some constant $\alpha>0$.

Hence by Lax-Milgram theorem, $a(\cdot, \cdot)$ gives rise to a bijective operator and by the compact embedding of $X_{0}$ in $\left(L^{2}(B)\right)^{3}$ and the fact that $G_{1 e}$ is a compact operator from $X_{0}$ into $H_{\text {div }}^{-1 / 2}\left(S_{R}\right)$, the second term $b(\cdot, \cdot)$ gives rise to a compact operator. Then a standard argument implies that the Fredholm alternative can be applied. Finally, the uniqueness theorem yields the existence result. We summarize the above analysis in the following theorem.

Theorem 2.6. For any incident field $E^{i}$, there exists a unique solution $w \in X$ of $(C B P)$ which depends continuously on the incident field $E^{i}$.

\section{Uniqueness for the Inverse Problem}

In this section we will show that the scattering obstacle $D$ and the corresponding parameter $\lambda$ are uniquely determined from the knowledge of the scattered fields $\left.E_{1, x_{0}}^{s}\left(x, x_{0}\right)\right|_{x \in \partial \Omega}$ for all $x_{0} \in S_{R_{0}}$, where $S_{R_{0}}$ is the surface of a large ball $B_{R_{0}}$ with $\bar{D} \subset \bar{\Omega} \subset B_{R_{0}}$. By some properties of the scattered fields, we can derive a relationship between them, then constructing special singular solutions which satisfy the relationship. Finally, we can obtain the uniqueness result by using the singularities of the singular solutions that we constructed.

Lemma 3.1. Assume that $k_{b}^{2}$ is not an eigenvalue of Maxwell equation for the domain $\Omega_{0}$. Then we have

(i) the restriction to $\partial \Omega_{0}$ of $\left\{v \times p\left(x_{0}\right) G\left(x, x_{0}\right): x_{0} \in S_{R_{0}}\right\}$ is complete in $H_{\text {div }}^{-1 / 2}\left(\partial \Omega_{0}\right)$;

(ii) the restriction to $\partial \Omega_{0}$ of $\left\{v \times \operatorname{curl}_{x} p\left(x_{0}\right) G\left(x, x_{0}\right)-i k \lambda p\left(x_{0}\right) G\left(x, x_{0}\right)_{T}: x_{0} \in S_{R_{0}}\right\}$ is complete in $L_{t}^{2}\left(\partial \Omega_{0}\right)$. 
Proof. For simplicity, we only prove statement (ii). Case (i) can be proved similarly.

Let $g \in L_{t}^{2}\left(\partial \Omega_{0}\right)$ be such that

$$
\int_{\partial \Omega_{0}}\left[v(x) \times \operatorname{curl}_{x} p\left(x_{0}\right) G\left(x, x_{0}\right)-i k \lambda p\left(x_{0}\right) G\left(x, x_{0}\right)_{T}\right] \cdot \bar{g}(x) d s(x)=0 .
$$

Then it follows that

$$
\int_{\partial \Omega_{0}}\left[\operatorname{curl}_{x_{0}}(\bar{g}(x) \times v(x)) G\left(x, x_{0}\right)-i k \lambda \bar{g}(x) G\left(x, x_{0}\right)\right] d s(x) \cdot p\left(x_{0}\right)=0 .
$$

Define

$$
\begin{gathered}
F_{1}^{*}\left(x_{0}\right)=\int_{\partial \Omega_{0}} \operatorname{curl}_{x_{0}}(\bar{g}(x) \times v(x)) G\left(x, x_{0}\right) d s(x), \\
F_{2}^{*}\left(x_{0}\right)=-\int_{\partial \Omega_{0}} i k \lambda \bar{g}(x) G\left(x, x_{0}\right) d s(x) \\
F^{*}\left(x_{0}\right)=F_{1}^{*}\left(x_{0}\right)+F_{2}^{*}\left(x_{0}\right) .
\end{gathered}
$$

By (3.2), it is easy to see that for arbitrary polarization $p\left(x_{0}\right)$ in the tangential plane to $S_{R_{0}}$ at $x_{0}$, we have

$$
F^{*}\left(x_{0}\right) \cdot p\left(x_{0}\right)=0, \quad \forall x_{0} \in S_{R_{0}}
$$

From the definition of (3.3), we immediately have

$$
\operatorname{curl}_{x_{0}} \operatorname{curl}_{x_{0}} F_{1}^{*}\left(x_{0}\right)-k_{b}^{2} F_{1}^{*}\left(x_{0}\right)=0, \quad \forall x_{0} \in \mathbb{R}^{3} \backslash \partial \Omega_{0} .
$$

Due to the symmetry of the background Green function, $F_{2}^{*}\left(x_{0}\right)$ as a function of $x_{0}$ solves $\operatorname{curl}_{x_{0}} \operatorname{curl}_{x_{0}} F_{2}^{*}\left(x_{0}\right)-k_{b}^{2} F_{2}^{*}\left(x_{0}\right)=0, \forall x_{0} \in \mathbb{R}^{3} \backslash \partial \Omega_{0}$. Hence, $F^{*}\left(x_{0}\right)$ satisfies the Maxwell's equation in $\mathbb{R}^{3} \backslash \partial \Omega_{0}$. By (3.6) and the fact that $p\left(x_{0}\right)$ is an arbitrary polarization in the tangential plane to $S_{R_{0}}$ at $x_{0}$, we immediately have that $v \times\left. F^{*}\left(x_{0}\right)\right|_{S_{R_{0}}}=0$.

The uniqueness of the exterior problem

$$
\begin{gathered}
\operatorname{curl}_{x_{0}} \operatorname{curl}_{x_{0}} F^{*}\left(x_{0}\right)-k_{b}^{2} F^{*}\left(x_{0}\right)=0, \text { in } \mathbb{R}^{3} \backslash \bar{B}_{R_{0}}, \\
v \times F^{*}\left(x_{0}\right)=0, \quad \text { on } S_{R_{0}}, \\
\lim _{\left|x_{0}\right| \rightarrow \infty}\left|x_{0}\right|\left(\operatorname{curl}_{x_{0}} F^{*}\left(x_{0}\right) \times v\left(x_{0}\right)-i k F^{*}\left(x_{0}\right)\right)=0
\end{gathered}
$$

implies that $F^{*}\left(x_{0}\right)=0$ in $\mathbb{R}^{3} \backslash \bar{B}_{R_{0}}$. Thus, the unique continuation principle ensures us that $F^{*}\left(x_{0}\right)=0$ in $\Omega_{0}^{e}=\mathbb{R}^{3} \backslash \bar{\Omega}_{0}$. By trace theorem, it follows that $v \times F^{*}\left(x_{0}\right)=0$ and $v \times \operatorname{curl}_{x_{0}} F^{*}\left(x_{0}\right)=$ 
0 on $\partial \Omega_{0}$. By the definition of $F^{*}\left(x_{0}\right)$ and the jump relations of the vector potential across $\partial \Omega_{0}$, it can be checked that $F^{*}\left(x_{0}\right)$ satisfies the following equations:

$$
\begin{aligned}
& \operatorname{curl}_{x_{0}} \operatorname{curl}_{x_{0}} F^{*}\left(x_{0}\right)-k_{b}^{2} F^{*}\left(x_{0}\right)=0, \quad \text { in } \Omega_{0}, \\
& v \times \operatorname{curl}_{x_{0}} F^{*}\left(x_{0}\right)-i k \lambda v \times F^{*}\left(x_{0}\right)=0, \quad \text { on } \partial \Omega_{0} \text {. }
\end{aligned}
$$

Therefore, the uniqueness theorem of the interior problem for Maxwell's equations implies that $F^{*}\left(x_{0}\right)=0$ in $\Omega_{0}$. Finally, from the jump relations of the vector potential across $\partial \Omega_{0}$, we have

$$
0=\left[\mathcal{v} \times F^{*}\left(x_{0}\right) \times\left. v\right|_{\partial \Omega_{0}}=-\bar{g}\left(x_{0}\right),\right.
$$

which completes the proof.

We now consider two obstacles $D_{1}$ and $D_{2}$ with the refractive index $n_{D_{j}}$ and the surface impedance $\lambda_{j}, j=1,2$. Let $U$ denote the unbounded part of $\mathbb{R}^{3} \backslash\left(\bar{D}_{1} \cup \bar{D}_{2}\right)$ and $D_{0}=\mathbb{R}^{3} \backslash \bar{U}$ its open complement. From the proof of Theorem 2.6, it follows that the total field $w_{j}, j=1,2$ satisfies

$$
\left(\operatorname{curl} w_{j}, \operatorname{curl} \phi\right)_{B_{R}}-\left(k_{j *}^{2} w_{j}, \phi\right)_{B_{R}}-i k\left\langle\lambda_{j} w_{j T}, \phi_{T}\right\rangle_{\Gamma_{j}}=-\left\langle v \times \operatorname{curl} w_{j}, \phi\right\rangle_{S_{R}}
$$

for any large ball $B_{R}$ with $D \subset B_{R}$ and all test function $\phi \in \tilde{X}$, where

$$
k_{j *}^{2}= \begin{cases}k_{D_{j^{\prime}}}^{2} & x \in D_{j} \\ k_{b^{\prime}}^{2} & x \in \mathbb{R}^{3} \backslash \bar{D}_{j} .\end{cases}
$$

It is convenient to introduce the following space:

$$
X_{j}=\left\{E \in H\left(\operatorname{curl}, B_{R}\right),\left.E_{T}\right|_{\Gamma_{j}} \in L_{t}^{2}\left(\Gamma_{j}\right)\right\}, \quad j=1,2,
$$

where $D_{j} \subset B_{R}$. The relationship derived in the following lemma plays a central role in the proof of the main result in this section.

Lemma 3.2. Assume that $k_{b}^{2}$ is not an eigenvalue of Maxwell equation in $\Omega$. Let $B_{R} \subset \mathbb{R}^{3}$ be a ball with $\bar{D}_{0} \subset \bar{\Omega} \subset B_{R}$. Let $E_{1, x_{0}}^{s}\left(x, x_{0}\right)$ and $E_{2, x_{0}}^{s}\left(x, x_{0}\right)$ be the scattered fields with respect to $D_{1}$ and $D_{2}$, respectively, produced by the same incident field $p G\left(\cdot, x_{0}\right)$. Assume that $v \times\left. E_{1, x_{0}}^{s}\right|_{\partial \Omega}=v \times\left. E_{2, x_{0}}^{s}\right|_{\partial \Omega}$ for all $x_{0} \in S_{R_{0}}$ with the radius $R_{0}>R$ for a fixed wave number $k$. Then we have

$$
\begin{aligned}
& k^{2}\left(\left(n_{D_{1}}-n_{b}\right) V_{1}, \bar{V}_{2}\right)_{D_{1}}+i k\left\langle\lambda_{1}\left(V_{1}\right)_{T},\left(\bar{V}_{2}\right)_{T}\right\rangle_{\Gamma_{1}} \\
& \quad=k^{2}\left(\left(n_{D_{2}}-n_{b}\right) V_{1}, \bar{V}_{2}\right)_{D_{2}}+i k\left\langle\lambda_{2}\left(V_{1}\right)_{T},\left(\bar{V}_{2}\right)_{T}\right\rangle_{\Gamma_{2}} .
\end{aligned}
$$


Here $V_{j} \in X_{1} \cap X_{2}$ satisfies the following variational problem:

$$
\left(a_{j} \operatorname{curl} V_{j}, \operatorname{curl} V\right)_{B_{R}}-\left(\kappa_{j} V_{j}, V\right)_{B_{R}}-i k\left\langle\tilde{\lambda}_{j}\left(V_{j}\right)_{T}, V_{T}\right\rangle_{\Gamma_{j}}=0,
$$

for all $V \in H_{0}$ (curl, $\left.B_{R}\right)$, where the coefficients $a_{j}, \kappa_{j} \in L^{\infty}\left(B_{R}\right)$ and $\tilde{\lambda}_{j} \in L^{\infty}\left(\Gamma_{j}\right)$ satisfy that $\left.a_{j}\right|_{D_{0}}=$ $1,\left.\kappa_{j}\right|_{D_{0}}=\left.\left(k_{j *}^{2}\right)\right|_{D_{0}}$ and $\left.\tilde{\lambda}_{j}\right|_{\Gamma_{j} \cap D_{0}}=\left.\lambda_{j}\right|_{\Gamma_{j}}, j=1,2$.

Proof. (i) We first prove that for any fixed $z \in U$, the scattered fields $E_{1, z}^{s}(x, z)=E_{2, z}^{s}(x, z)$, where $\left(V_{j, z}, E_{j, z}^{s}\right), j=1,2$ is the solution of the following problem:

$$
\begin{gathered}
\text { curlcurl } V-k_{D_{j}}^{2} V=0, \quad \text { in } D_{j}, \\
\operatorname{curlcurl} E^{s}-k_{b}^{2} E^{s}=0, \quad \text { in } \mathbb{R}^{3} \backslash \bar{D}_{j}, \\
v \times\left(V-E^{s}\right)=f, \quad \text { on } \Gamma_{j}, \\
v \times \operatorname{curl}\left(V-E^{s}\right)=-i k \lambda_{j} E_{T}^{s}+r+h, \text { on } \Gamma_{j},
\end{gathered}
$$

with the incident field $E^{i}=p G(\cdot, z)$ and $f=v \times E^{i}, h=v \times \operatorname{curl} E^{i}, r=-i k \lambda_{j} E_{T}^{i}$. By Lemma 3.1 and the fact that $k_{b}^{2}$ is not an eigenvalue of Maxwell equation in $\Omega$, it follows that there exists a sequence $a_{n} \in \mathbb{R}$ and $x_{0}^{(n)} \in S_{R_{0}}$ such that

$$
\left\|v \times p G(\cdot, z)-\sum_{n} a_{n} v \times p G\left(\cdot, x_{0}^{(n)}\right)\right\|_{H_{\text {div }}^{-1 / 2}(\partial \Omega)}<\varepsilon, \quad \forall \varepsilon>0 .
$$

Let $E(x)=p G(\cdot, z)-\sum_{n} a_{n} p G\left(\cdot, x_{0}^{(n)}\right)$, then it satisfies the Maxwell equation curl curl $E-k_{b}^{2} E=$ 0 in $\Omega \backslash\{z\}$. Let $f=\left.(v \times E)\right|_{\partial \Omega}$, then the well posedness of the problem

$$
\begin{gathered}
\operatorname{curl} \operatorname{curl} E-k_{b}^{2} E=0, \quad \text { in } \Omega \backslash\{z\}, \\
v \times E=f, \quad \text { on } \partial \Omega
\end{gathered}
$$

and (3.17) imply that

$$
\|E\|_{H(\operatorname{curl}, \Omega)} \leq C_{1}\|v \times E\|_{H_{\text {div }}^{-1 / 2}(\partial \Omega)}<C_{1} \varepsilon, \quad \forall \varepsilon>0
$$

This, together with the fact that $\operatorname{div} E=0$, implies (see [28])

$$
\|E\|_{H^{1}(\Omega)} \leq C_{2}\left(\|E\|_{H(\operatorname{curl}, \Omega)}+\|E\|_{H(\operatorname{div}, \Omega)}+\|v \times E\|_{H_{\text {div }}^{-1 / 2}(\partial \Omega)}\right) \leq C_{2} \varepsilon, \quad \forall \varepsilon>0 .
$$


Then by (3.19), (3.20), and the trace theorem, it can be proved that

$$
\begin{aligned}
\| v \times & E\left\|_{H_{\text {div }}^{-1 / 2}\left(\Gamma_{j}\right)}+\right\| v \times \operatorname{curl} E\left\|_{L_{t}^{2}\left(\Gamma_{j}\right)}+\right\| v \times \operatorname{curl} E-i k \lambda_{j} E_{T} \|_{L_{t}^{2}\left(\Gamma_{j}\right)} \\
& \leq C_{3}\left(\|E\|_{H\left(\operatorname{curl}, D_{j}\right)}+\left\|E_{T}\right\|_{L_{t}^{2}\left(\Gamma_{j}\right)}\right) \leq C_{4}\left(\|E\|_{H\left(\operatorname{curl}, D_{j}\right)}+\|E\|_{H^{1}\left(D_{j}\right)}\right) \\
& \leq C_{5}\left(\|E\|_{H(\operatorname{curl}, \Omega)}+\|E\|_{H^{1}(\Omega)}\right) \leq C_{5} \varepsilon, \quad j=1,2, \forall \varepsilon>0 .
\end{aligned}
$$

Denote by $\widetilde{E}_{1}^{s}$ and $\widetilde{E}_{2}^{s}$ the scattered fields with respect to $D_{1}$ and $D_{2}$ produced by the same incident field $\sum_{n} a_{n} p G\left(\cdot, x_{0}^{(n)}\right)$. By the assumption $v \times\left. E_{1, x_{0}}^{s}\right|_{\partial \Omega}=v \times\left. E_{2, x_{0}}^{s}\right|_{\partial \Omega}$ for all $x_{0} \in S_{R_{0}}$, it is easy to see that $v \times\left.\widetilde{E}_{1}^{s}\right|_{\partial \Omega}=v \times\left.\widetilde{E}_{2}^{s}\right|_{\partial \Omega}$. Then by the uniqueness theorem of the exterior scattering problem, it follows that $\widetilde{E}_{1}^{s}=\widetilde{E}_{2}^{s}$ in $\mathbb{R}^{3} \backslash \bar{\Omega}$, which together with the unique continuation principle ensures that $\widetilde{E}_{1}^{s}=\widetilde{E}_{2}^{s}$ in $U$. Now, by (3.21) and the well posedness of the direct problem (2.18), it can be checked that for any compact set $K \subset \subset U$, we have

$$
\begin{aligned}
& \left\|E_{1, z}^{s}(x, z)-\widetilde{E}_{1}^{s}(x)\right\|_{H(\operatorname{curl}, K)}<\varepsilon, \quad \forall \varepsilon>0, \\
& \left\|E_{1, z}^{s}(x, z)-\widetilde{E}_{2}^{s}(x)\right\|_{H(\operatorname{curl}, K)}<\varepsilon, \quad \forall \varepsilon>0,
\end{aligned}
$$

for any fixed $z \in K$.

Therefore, the fact $\widetilde{E}_{1}^{s}=\widetilde{E}_{2}^{s}$ in $U$ ensures us that

$$
\left\|E_{1, z}^{s}(x, z)-E_{2, z}^{S}(x, z)\right\|_{H\left(\operatorname{curl}_{, K}\right)}<\varepsilon, \quad \forall \varepsilon>0 .
$$

The arbitrarity of $\varepsilon$ implies that $E_{1, z}^{s}(x, z)=E_{2, z}^{s}(x, z)$ for any fixed $z \in K$.

(ii) Next we will show that the identity (3.14) holds. Set $w^{*}=w_{2}-w_{1}$, then it follows from (3.11) that

$$
\begin{aligned}
& \left(\operatorname{curl} w^{*}, \operatorname{curl} V\right)_{B_{R}}-\left(k_{2 *}^{2} w^{*}, V\right)_{B_{R}}-i k\left\langle\lambda_{2} w_{T}^{*}, V_{T}\right\rangle_{\Gamma_{2}} \\
& =-\left(\left(k_{1 *}^{2}-k_{2 *}^{2}\right) w_{1}, V\right)_{B_{R}}-i k\left\langle\lambda_{1}\left(w_{1}\right)_{T}, V_{T}\right\rangle_{\Gamma_{1}}+i k\left\langle\lambda_{2}\left(w_{1}\right)_{T}, V_{T}\right\rangle_{\Gamma_{2}}
\end{aligned}
$$

for all $V \in X^{*}=\left\{V \in X_{1} \cap X_{2},\left.V_{T}\right|_{S_{R}}=0\right\}$. Choose two domains $\Omega_{1}, \Omega_{2} \subset B_{R}$ with $\bar{D}_{0} \subset \Omega_{1} \subset \Omega_{2}$ and define a smooth function $\psi \in C^{\infty}\left(\mathbb{R}^{3}\right)$ with $\psi=1$ in $\Omega_{1}$ and $\psi=0$ in $\mathbb{R}^{3} \backslash \Omega_{2}$. Let $a_{2}=\psi$, $\kappa_{2}=\psi k_{2 *^{\prime}}^{2}, \tilde{\lambda}_{2}=\lambda_{2}$, it is easy to see that $a_{2}, \kappa_{2}, \tilde{\lambda}_{2}$ satisfy the assumptions of the lemma. We further assume $V_{2}$ satisfies (3.15) with respect to $a_{2}=\psi, \kappa_{2}=\psi k_{2 *^{\prime}}^{2} \tilde{\lambda}_{2}=\lambda_{2}$, so that substituting $V=\overline{\psi V_{2}}$ into the left hand of (3.24) and noting that $w^{*}=0$ in $U$ yield that

$$
\begin{aligned}
& \left(\operatorname{curl} w^{*}, \operatorname{curl}\left(\overline{\psi V_{2}}\right)\right)_{B_{R}}-\left(k_{2 *}^{2} w^{*}, \overline{\psi V_{2}}\right)_{B_{R}}-i k\left\langle\lambda_{2} w_{T}^{*}, \overline{\psi\left(V_{2}\right)_{T}}\right\rangle_{\Gamma_{2}} \\
& =-\left(a_{2} \operatorname{curl} V_{2}, \operatorname{curl} \bar{w}^{*}\right)_{B_{R}}-\left(\kappa_{2} V_{2}, \bar{w}^{*}\right)_{B_{R}}-i k\left\langle\tilde{\lambda}_{2} V_{2 T}, \bar{w}_{T}^{*}\right\rangle_{\Gamma_{2}}=0 .
\end{aligned}
$$


Hence substituting $V=\overline{\psi V_{2}}$ into (3.24), it follows from the right hand of (3.24) that

$$
-\left(\left(k_{1 *}^{2}-k_{2 *}^{2}\right) w_{1}, \bar{V}_{2}\right)_{B_{R}}-i k\left\langle\lambda_{1}\left(w_{1}\right)_{T},\left(\bar{V}_{2}\right)_{T}\right\rangle_{\Gamma_{1}}+i k\left\langle\lambda_{2}\left(w_{1}\right)_{T},\left(\bar{V}_{2}\right)_{T}\right\rangle_{\Gamma_{2}}=0
$$

We define $f \in X_{1}$ by

$$
(f, \phi)_{X_{1}}=-\left(\left(k_{1 *}^{2}-k_{2 *}^{2}\right) V_{2}, \phi\right)_{B_{R}}-i k\left\langle\lambda_{1}\left(V_{2}\right)_{T}, \phi_{T}\right\rangle_{\Gamma_{1}}+i k\left\langle\lambda_{2}\left(V_{2}\right)_{T}, \phi_{T}\right\rangle_{\Gamma_{2}}
$$

for all $\phi \in X_{1}$. By Theorem 2.6, it follows that there exists a unique solution $w_{0} \in X_{1}$ of the problem

$$
\left(\operatorname{curl} w_{0}, \operatorname{curl} \phi\right)_{B_{R}}-\left(k_{1 *}^{2} w_{0}, \phi\right)_{B_{R}}-i k\left\langle\lambda_{1}\left(w_{0}\right)_{T}, \phi_{T}\right\rangle_{\Gamma_{1}}+i k\left\langle G e\left(v \times w_{0}\right), \phi_{T}\right\rangle_{S_{R}}=(f, \phi)_{X_{1}}
$$

for all $\phi \in X_{1}$. Choose two domains $\Omega_{e}, \Omega_{i} \subseteq B_{R}$ with $\bar{\Omega} \subseteq \Omega_{e}, \bar{D}_{0} \subset \Omega_{i} \subset \Omega$ and define smooth functions $\psi_{e}, \psi_{i} \in C^{\infty}\left(\mathbb{R}^{3}\right)$ with $\psi_{e}=1, x \in \Omega, \psi_{e}=0, x \in \mathbb{R}^{3} \backslash \Omega_{e}$ and $\psi_{i}=1, x \in$ $\Omega_{i}, \psi_{i}=0, x \in \mathbb{R}^{3} \backslash \Omega$. Take $j=1, \phi=\overline{\psi_{e} w_{0}}$ in (3.11), it is seen that

$$
\left(\operatorname{curl} w_{1}, \operatorname{curl}\left(\overline{\psi_{e} w_{0}}\right)\right)_{B_{R}}-\left(k_{1 *}^{2} w_{1}, \overline{\psi_{e} w_{0}}\right)_{B_{R}}-i k\left\langle\lambda_{1}\left(w_{1}\right)_{T},\left(\bar{w}_{0}\right)_{T}\right\rangle_{\Gamma_{1}}=0
$$

Equation (3.28) with $\phi$ replaced by $\overline{\psi_{i} w_{1}}$ yields

$$
\left(\operatorname{curl} w_{0}, \operatorname{curl}\left(\overline{\psi_{i} w_{1}}\right)\right)_{B_{R}}-\left(k_{1 *}^{2} w_{0}, \overline{\psi_{i} w_{1}}\right)_{B_{R}}-i k\left\langle\lambda_{1}\left(w_{0}\right)_{T},\left(\bar{w}_{1}\right)_{T}\right\rangle_{\Gamma_{1}}=\left(f, \overline{\psi_{i} w_{1}}\right)_{X_{1}}
$$

By (3.26) and (3.27), it can be shown that

$$
\begin{aligned}
\left(f, \overline{\varphi_{i} w_{1}}\right)_{X_{1}}= & -\left(\left(k_{1 *}^{2}-k_{2 *}^{2}\right) V_{2}, \bar{w}_{1}\right)_{B_{R}}-i k\left\langle\lambda_{1}\left(V_{2}\right)_{T},\left(\bar{w}_{1}\right)_{T}\right\rangle_{\Gamma_{1}} \\
& +i k\left\langle\lambda_{2}\left(V_{2}\right)_{T},\left(\bar{w}_{1}\right)_{T}\right\rangle_{\Gamma_{2}}=0 .
\end{aligned}
$$

Taking the difference of (3.29) and (3.30), we have that

$$
\begin{aligned}
0= & \int_{\Omega \backslash \Omega_{i}} \operatorname{curl} w_{0} \cdot \operatorname{curl}\left(\psi_{i} w_{1}-w_{1}\right)-k_{b}^{2} w_{0} \cdot\left(\psi_{i} w_{1}-w_{1}\right) d x \\
& -\int_{\Omega_{e} \backslash \Omega} \operatorname{curl}\left(\psi_{e} w_{0}\right) \cdot \operatorname{curl} w_{1}-k_{b}^{2}\left(\psi_{e} w_{0}\right) \cdot w_{1} d x
\end{aligned}
$$

By (3.28), we can deduce that $w_{0}$ is a radiating solution of the corresponding Maxwell's equations in $B_{R}$, then it can be extended to all of $\mathbb{R}^{3}$ denoted by $w_{0}^{e}$ by solving the exterior 
Maxwell's equation in $\mathbb{R}^{3} \backslash \bar{B}_{R}$ with $v \times w_{0}^{e}=v \times w_{0}$ on $S_{R}$, which also satisfies the SilverMüler radiation condition at infinity. By applying the vector Green formula to (3.32), it can be proved that

$$
0=\int_{\partial \Omega}\left[w_{1} \cdot\left(v \times \operatorname{curl} w_{0}^{e}\right)-w_{0}^{e} \cdot\left(v \times \operatorname{curl} w_{1}\right)\right] d s .
$$

In view of the fact $w_{1}=E_{1}=E_{1}^{S}+E^{i}$ and $E^{i}=p G\left(\cdot, x_{0}\right)$ in $\mathbb{R}^{3} \backslash \bar{D}_{1}$, we immediately have

$$
\begin{aligned}
0= & \int_{\partial \Omega} E_{1}^{s} \cdot\left(v \times \operatorname{curl} w_{0}^{e}\right)-w_{0}^{e} \cdot\left(v \times \operatorname{curl} E_{1}^{s}\right) d s \\
& +\int_{\partial \Omega}\left[\left(v \times \operatorname{curl} w_{0}^{e}\right) \cdot p G\left(\cdot, x_{0}\right)-w_{0}^{e} \cdot\left(v \times \operatorname{curl}\left(p G\left(\cdot, x_{0}\right)\right)\right)\right] d s .
\end{aligned}
$$

Application of the vector Green formula again and noting that both $E_{1}^{s}$ and the extended function $w_{0}^{e}$ satisfy the Silver-Müler radiation condition, it follows that

$$
\begin{aligned}
\int_{\partial \Omega} & E_{1}^{s} \cdot\left(v \times \operatorname{curl} w_{0}^{e}\right)-w_{0}^{e} \cdot\left(v \times \operatorname{curl} E_{1}^{s}\right) d s \\
\quad & \int_{S_{R}} E_{1}^{s} \cdot\left(v \times \operatorname{curl} w_{0}^{e}\right)-w_{0}^{e} \cdot\left(v \times \operatorname{curl} E_{1}^{s}\right) d s=0 .
\end{aligned}
$$

Hence the Stratton-Chu formula combines with (3.34) implies that

$$
0=p\left(x_{0}\right) \cdot w_{0}^{e}\left(x_{0}\right), \quad \forall x_{0} \in S_{R_{0}}, \forall p\left(x_{0}\right) \in \mathbb{R}^{3} .
$$

Since $p\left(x_{0}\right)$ is an arbitrary polarization in the tangential plane to $S_{R_{0}}$ at $x_{0}$, we obtain that $v \times\left. w_{0}^{e}\left(x_{0}\right)\right|_{S_{R_{0}}}=0$. By the fact that $w_{0}^{e}$ is a radiating solution of Maxwell's equation in $\mathbb{R}^{3} \backslash \bar{B}_{R_{0}}$, it follows that $w_{0}^{e}=0$ in $\mathbb{R}^{3} \backslash \bar{B}_{R_{0}}$. Hence the unique continuation principle implies that $w_{0}^{e}=0$ in $\mathbb{R}^{3} \backslash \bar{U}$. Therefore, $w_{0}^{e}$ can be used as a test function for $V_{1}$, which satisfies (3.15) with $a_{1}=\psi_{i}, \kappa_{1}=\psi_{i} k_{1 *^{\prime}}^{2}, \tilde{\lambda}_{1}=\lambda_{1}$. So that from the left hand of (3.30), we deduce that

$$
\begin{aligned}
& \left(\operatorname{curl} w_{0}^{e}, \operatorname{curl}\left(\overline{\psi_{i} V_{1}}\right)\right)_{B_{R}}-\left(k_{1 *}^{2} w_{0}^{e}, \overline{\psi_{i} V_{1}}\right)_{B_{R}}-i k\left\langle\lambda_{1}\left(w_{0}^{e}\right)_{T},\left(\bar{V}_{1}\right)_{T}\right\rangle_{\Gamma_{1}} \\
& =\left(a_{1} \operatorname{curl} V_{1}, \operatorname{curl} \bar{w}_{0}^{e}\right)_{B_{R}}-\left(\kappa_{1} V_{1}, \bar{w}_{0}^{e}\right)_{B_{R}}-i k\left\langle\tilde{\lambda}_{1}\left(V_{1}\right)_{T},\left(\bar{w}_{0}^{e}\right)_{T}\right\rangle_{\Gamma_{1}}=0 .
\end{aligned}
$$

Thus, it follows from the right hand of (3.30) that $\left(f, \overline{\psi_{i} V_{1}}\right)_{X_{1}}=0$. Furthermore, from (3.27) with $\phi$ replaced by $\overline{\psi_{i} V_{1}}$, it can be shown that

$$
-\left(\left(k_{1 *}^{2}-k_{2 *}^{2}\right) V_{2}, V_{1}\right)_{B_{R}}-i k\left\langle\left\langle\lambda_{1}\left(V_{1}\right)_{T},\left(V_{2}\right)_{T}\right\rangle_{\Gamma_{1}}+i k\left\langle\lambda_{2}\left(V_{2}\right)_{T},\left(V_{1}\right)_{T}\right\rangle_{\Gamma_{2}}=0\right.
$$


From the definitions of $k_{j *^{\prime}}^{2}$ we observe that

$$
k_{1 *}^{2}-k_{2 *}^{2}= \begin{cases}k^{2} n_{D_{1}}-k^{2} n_{b}, & x \in D_{1}, \\ k^{2} n_{D_{2}}-k^{2} n_{b}, & x \in D_{2}, \\ 0, & x \in B_{R} \backslash\left(\bar{D}_{1} \cup \bar{D}_{2}\right),\end{cases}
$$

which combines (3.38), the definition of the scalar product $(\cdot, \cdot)_{D}$, and the fact that $\left(\left(k_{1 *}^{2}-k_{2 *}^{2}\right) V_{2}, V_{1}\right)_{B_{R}}=k^{2}\left(\left(n_{D_{1}}-n_{b}\right) V_{2}, V_{1}\right)_{D_{1}}-k^{2}\left(\left(n_{D_{2}}-n_{b}\right) V_{2}, V_{1}\right)_{D_{2}}$ implies that (3.14) holds. This ends the proof of this lemma.

The main result of this section is contained in the following theorem.

Theorem 3.3. Let $E_{1}^{s}$ and $E_{2}^{s}$ be the scattered fields with respect to $D_{1}$ and $D_{2}$, respectively, and $\lambda_{1}, \lambda_{2}$ the corresponding impedances. Suppose that the assumptions in Lemma 3.2 hold true and $\Gamma_{j} \cap\left(D_{i} \backslash D_{j}\right)$ is not empty for $i, j=1,2, i \neq j$. If one of the following assumptions holds, then we have $D_{1}=D_{2}$. Consider

(i) $\operatorname{Re} \lambda_{j} \geq \delta>0$;

(ii) $\operatorname{Im} \lambda_{j} \geq \delta>0$ or $\operatorname{Im} \lambda_{j} \leq-\delta<0$.

Proof. Let us assume that $\bar{D}_{1}$ is not included in $\bar{D}_{2}$. Since $D_{2}^{e}=\mathbb{R}^{3} \backslash \bar{D}_{2}$ is connected, we can find a point $z \in \Gamma_{1} \backslash D_{2}$ and a sufficiently small $\varepsilon>0$ with the following properties:

(i) $B_{2 \varepsilon}(z) \cap \bar{D}_{2}=\emptyset$;

(ii) the points $z_{n}=z+(\varepsilon / n) \mathcal{v}(z)$ lie in $B_{2 \varepsilon}(z)$ for all $n \in N$, where $v(z)$ is the unit normal to $\Gamma_{1}$ at $z$.

Denote $D=\left(\bar{D}_{1} \backslash \bar{D}_{2}\right)^{\circ}$, the inner part of the domain $\bar{D}_{1} \backslash \bar{D}_{2}$. We consider the unique solution of the following problem:

$$
\begin{gathered}
\operatorname{curl} \operatorname{curl} V-k_{D}^{2} V=0, \quad \text { in } D, \\
\operatorname{curl} \operatorname{curl} E^{s}-k_{b}^{2} E^{S}=0, \quad \text { in } \mathbb{R}^{3} \backslash \bar{D}, \\
v \times\left(E^{S}-V\right)=-v \times E_{m}\left(\cdot, z_{n}\right), \quad \text { on } \partial D, \\
v \times \operatorname{curl}\left(E^{S}-V\right)=i k \lambda_{1}\left(E_{T}^{S}+E_{m}\left(\cdot, z_{n}\right)\right)-v \times \operatorname{curl} E_{m}\left(\cdot, z_{n}\right), \quad \text { on } \Gamma_{1} \cap \partial D .
\end{gathered}
$$

Here $E^{s}$ satisfies the Silver-Müler radiation condition at infinity, and $E_{m}$ denotes the magnetic dipole defined by

$$
E_{m}\left(x, z_{n}\right)=\operatorname{curl}_{x}\left(v(z) \frac{e^{i k_{b}\left|x-z_{n}\right|}}{\left|x-z_{n}\right|}\right)
$$


Define

$$
F_{1}\left(x, z_{n}\right)= \begin{cases}V(x), & \text { if } x \in D \\ E^{S}(x)+E_{m}\left(x, z_{n}\right), & \text { if } x \in \mathbb{R}^{3} \backslash\left(\bar{D} \cup z_{n}\right)\end{cases}
$$

It can be proved that $F_{1}\left(x, z_{n}\right)$ is a solution of Maxwell's equations with homogeneous conductive boundary value conditions on $\partial D$ in any domain $\Omega \subset \mathbb{R}^{3}$ with $\bar{D}_{0} \subset \Omega$ and $z_{n} \nsubseteq \Omega$.

Define

$$
\kappa_{1}(x)= \begin{cases}k_{1 *}^{2}(x) & \text { if } x \in \mathbb{R}^{3} \backslash\left(\overline{D_{1} \backslash D}\right), \\ k_{b}^{2} & \text { if } x \in D_{1} \backslash D\end{cases}
$$

and $\tilde{\lambda}_{1}(x)=\lambda_{1}$.

In view of the above definitions of $\kappa_{1}(x)$ and $\tilde{\lambda}_{1}(x)$, it follows that $F_{1}\left(x, z_{n}\right)$ satisfies the variational equation (3.15) in Lemma 3.2 for the obstacle $D$. The well posedness of the direct problem for (CBP) and the fact that $z_{n}$ is bounded away from $\partial D$ imply that the solution $\left(V, E^{s}\right)$ of $(3.40)$ is uniformly bounded in $\tilde{X}$. We now define another singular solution with respect to $D_{2}$ by

$$
F_{2}\left(x, z_{n}\right)= \begin{cases}\tilde{V}(x), & \text { if } x \in D_{2}, \\ \tilde{E}^{s}(x)+E_{m}\left(x, z_{n}\right), & \text { if } x \in \mathbb{R}^{3} \backslash\left(\bar{D}_{2} \cup z_{n}\right),\end{cases}
$$

where $E_{m}$ is a magnetic dipole defined in (3.41), and $\left(\tilde{V}, \widetilde{E}^{s}\right)$ is a solution of the problem

$$
\begin{gathered}
\text { curl curl } \tilde{V}-k_{D_{2}}^{2} \tilde{V}=0, \quad \text { in } D_{2}, \\
\operatorname{curl} \operatorname{curl} \widetilde{E}^{s}-k_{b}^{2} \widetilde{E}^{s}=0, \quad \text { in } \mathbb{R}^{3} \backslash\left(\bar{D}_{2} \cup z_{n}\right), \\
v \times\left(\widetilde{E}^{s}-\tilde{V}\right)=-v \times E_{m}\left(\cdot, z_{n}\right), \quad \text { on } \partial D, \\
v \times \operatorname{curl}\left(\tilde{E}^{s}-\tilde{V}\right)=i k \lambda_{2}\left(\tilde{E}_{T}^{s}+\left(E_{m}\left(\cdot, z_{n}\right)\right)_{T}\right)-v \times \operatorname{curl} E_{m}\left(\cdot, z_{n}\right), \quad \text { on } \Gamma_{2} .
\end{gathered}
$$

Here $\widetilde{E}^{s}$ satisfies the Silver-Müler radiation condition at infinity. Noting that $F_{2}\left(x, z_{n}\right)$ satisfies the variational equation (3.15) in Lemma 3.2 with $\kappa_{2}(x)=k_{2 *}^{2}$ and $\tilde{\lambda}_{2}=\lambda_{2}$, it follows that both $F_{1}\left(x, z_{n}\right)$ and $F_{2}\left(x, z_{n}\right)$ satisfy the relationship (3.14), then we obtain

$$
\begin{aligned}
& k^{2}\left(\left(n_{D}-n_{b}\right) F_{1}, \bar{F}_{2}\right)_{D}+i k\left\langle\lambda_{1}\left(F_{1}\right)_{T},\left(\bar{F}_{2}\right)_{T}\right\rangle_{\partial D} \\
& \quad=k^{2}\left(\left(n_{D_{2}}-n_{b}\right) F_{1}, \bar{F}_{2}\right)_{D_{2}}+i k\left\langle\lambda_{2}\left(F_{2}\right)_{T},\left(\bar{F}_{1}\right)_{T}\right\rangle_{\Gamma_{2}} .
\end{aligned}
$$

For case (i), by the fact that $z \in \Gamma_{1} \backslash D_{2}$ and the singularities of the magnetic dipole $E_{m}$ defined in (3.41), it can be proved that $\left|k^{2}\left(\left(n_{D}-n_{b}\right) F_{1}, \bar{F}_{2}\right)_{D}+i k\left\langle\lambda_{1}\left(F_{1}\right)_{T},\left(\bar{F}_{2}\right)_{T}\right\rangle_{\partial D}\right| \rightarrow \infty$ as $n \rightarrow \infty$, 
this, together with the fact that the other terms in the right hand of (3.46) are bounded, leads to a contradiction. Hence we have $D_{1} \subset D_{2}$. By choosing $z \in \Gamma_{2} \backslash D_{1}$ and using the similar analysis as in the proof above, one can prove that $D_{2} \subset D_{1}$. Finally, we obtain that $D_{1}=D_{2}$. For other cases, due to the singularities of $F_{j}(j=1,2)$, a contradiction also arises in (3.46) as $n \rightarrow \infty$. This proves the theorem.

Theorem 3.4. Assume $D_{1}=D_{2}$ with parameters $\lambda_{j} \in C\left(\partial \bar{D}_{j}\right)$ and the scattered fields $E_{j, x_{0}}^{s}$ ( $j=$ $1,2)$ satisfy $v \times\left. E_{1, x_{0}}^{s}\right|_{\partial \Omega}=v \times E_{2, x_{0}}^{s} \mid \partial \Omega$ for all $x_{0} \in S_{R_{0}}$, then we have $\lambda_{1}=\lambda_{2}$ on $\partial D$.

Proof. From the proof of Theorem 3.3, it follows that there exists two singular solutions $F_{1}, F_{2}$ of the conductive boundary problem with respect to the obstacle $D$ for some $z \in \partial D$. By Lemma 3.2 and the identity $D_{1}=D_{2}$, it can be checked that

$$
\int_{D}\left(k_{1 *}^{2}-k_{2 *}^{2}\right) F_{1}\left(x, z_{n}\right) \cdot F_{2}\left(x, z_{n}\right) d x+i k \int_{\partial D}\left(\lambda_{1}-\lambda_{2}\right) F_{1}\left(x, z_{n}\right) \cdot F_{2}\left(x, z_{n}\right) d s(x)=0
$$

The singularities of $F_{1}, F_{2}$ ensure that $\lambda_{1}=\lambda_{2}$. This completes the proof of the theorem.

\section{Acknowledgments}

This work was supported by the NNSF of China under Grant $(11201402,11201266)$ and Tianyuan Youth Foundations for Mathematics of NNSFC (Grant no. 11026098, 11026150).

\section{References}

[1] F. Hettlich, "Uniqueness of the inverse conductive scattering problem for time-harmonic electromagnetic waves," SIAM Journal on Applied Mathematics, vol. 56, no. 2, pp. 588-601, 1996.

[2] T. S. Angell and A. Kirsch, "The conductive boundary condition for Maxwell's equations," SIAM Journal on Applied Mathematics, vol. 52, no. 6, pp. 1597-1610, 1992.

[3] G. Vasseur and P. Weidelt, "Bimodal electromagnetic induction in nonuniform thin sheets with an application of the northern Pyrenean induction anomaly," Geophysical Journal of the Royal Astronomical Society, vol. 51, pp. 669-690, 1977.

[4] F. Cakoni, D. Colton, and P. Monk, "The determination of the surface conductivity of a partially coated dielectric," SIAM Journal on Applied Mathematics, vol. 65, no. 3, pp. 767-789, 2005.

[5] F. Cakoni, D. Colton, and P. Monk, "The inverse electromagnetic scattering problem for a partially coated dielectric," Journal of Computational and Applied Mathematics, vol. 204, no. 2, pp. 256-267, 2007.

[6] F. Cakoni, D. Colton, and P. Monk, "The electromagnetic inverse-scattering problem for partly coated Lipschitz domains," Proceedings of the Royal Society of Edinburgh A, vol. 134, no. 4, pp. 661-682, 2004.

[7] F. Cakoni and H. Haddar, "Identification of partially coated anisotropic buried objects using electromagnetic Cauchy data," Journal of Integral Equations and Applications, vol. 19, no. 3, pp. 359$389,2007$.

[8] V. Isakov, "On uniqueness of recovery of a discontinuous conductivity coefficient," Communications on Pure and Applied Mathematics, vol. 41, no. 7, pp. 865-877, 1988.

[9] V. Isakov, "On uniqueness in the inverse transmission scattering problem," Communications in Partial Differential Equations, vol. 15, no. 11, pp. 1565-1587, 1990.

[10] A. Kirsch and R. Kress, "Uniqueness in inverse obstacle scattering," Inverse Problems, vol. 9, no. 2, pp. 285-299, 1993.

[11] T. Gerlach and R. Kress, "Uniqueness in inverse obstacle scattering with conductive boundary condition," Inverse Problems, vol. 12, no. 5, pp. 619-625, 1996. 
[12] D. Mitrea and M. Mitrea, "Uniqueness for inverse conductivity and transmission problems in the class of Lipschitz domains," Communications in Partial Differential Equations, vol. 23, no. 7-8, pp. 1419-1448, 1998.

[13] N. Valdivia, "Uniqueness in inverse obstacle scattering with conductive boundary conditions," Applicable Analysis, vol. 83, no. 8, pp. 825-851, 2004.

[14] M. Di Cristo and J. Sun, "An inverse scattering problem for a partially coated buried obstacle," Inverse Problems, vol. 22, no. 6, pp. 2331-2350, 2006.

[15] M. Di Cristo and J. Sun, "The determination of the support and surface conductivity of a partially coated buried object," Inverse Problems, vol. 23, no. 3, pp. 1161-1179, 2007.

[16] G. Yan and P. Y. H. Pang, "Inverse obstacle scattering with two scatterers," Applicable Analysis, vol. 70, no. 1-2, pp. 35-43, 1998.

[17] X. Liu and B. Zhang, "Inverse scattering by an inhomogeneous penetrable obstacle in a piecewise homogeneous medium," Acta Mathematica Scientia B, vol. 32, no. 4, pp. 1281-1297, 2012.

[18] X. Liu, B. Zhang, and G. Hu, "Uniqueness in the inverse scattering problem in a piecewise homogeneous medium," Inverse Problems, vol. 26, no. 1, p. 015002, 14, 2010.

[19] X. Liu and B. Zhang, "Direct and inverse obstacle scattering problems in a piecewise homogeneous medium," SIAM Journal on Applied Mathematics, vol. 70, no. 8, pp. 3105-3120, 2010.

[20] P. Hähner, "A uniqueness theorem for a transmission problem in inverse electromagnetic scattering," Inverse Problems, vol. 9, no. 6, pp. 667-678, 1993.

[21] X. Liu, B. Zhang, and J. Yang, "The inverse electromagnetic scattering problem in a piecewise homogeneous medium," Inverse Problems, vol. 26, no. 12, p. 125001, 19, 2010.

[22] D. Colton, R. Kress, and P. Monk, "Inverse scattering from an orthotropic medium," Journal of Computational and Applied Mathematics, vol. 81, no. 2, pp. 269-298, 1997.

[23] M. Piana, "On uniqueness for anisotropic inhomogeneous inverse scattering problems," Inverse Problems, vol. 14, no. 6, pp. 1565-1579, 1998.

[24] P. Hähner, "On the uniqueness of the shape of a penetrable, anisotropic obstacle," Journal of Computational and Applied Mathematics, vol. 116, no. 1, pp. 167-180, 2000.

[25] F. Cakoni and D. Colton, "A uniqueness theorem for an inverse electromagnetic scattering problem in inhomogeneous anisotropic media," Proceedings of the Edinburgh Mathematical Society II, vol. 46, no. 2, pp. 293-314, 2003.

[26] D. Colton and R. Kress, Inverse Acoustic and Electromagnetic Scattering Theory, vol. 93, Springer, New York, NY, USA, 2nd edition, 1998.

[27] P. Monk, Finite Element Methods for Maxwell's Equations, Oxford University Press, Oxford, UK, 2003.

[28] M. Cessenat, Mathematical Methods in Electromagnetism, vol. 41, World Scientific Publishing, Singapore, 1996. 


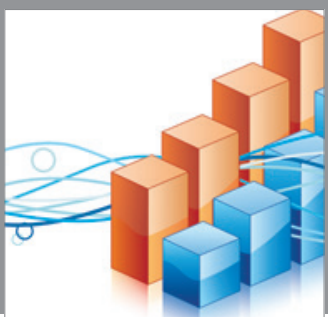

Advances in

Operations Research

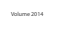

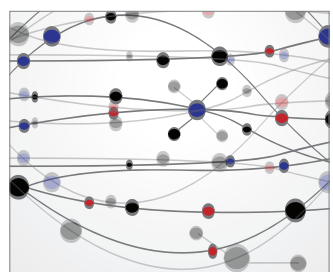

\section{The Scientific} World Journal
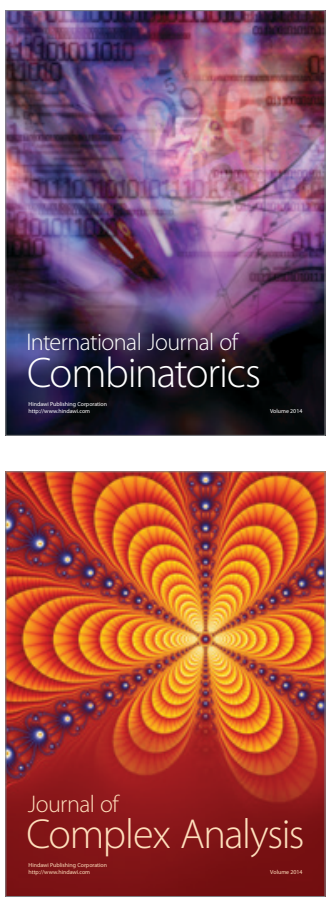

International Journal of

Mathematics and

Mathematical

Sciences
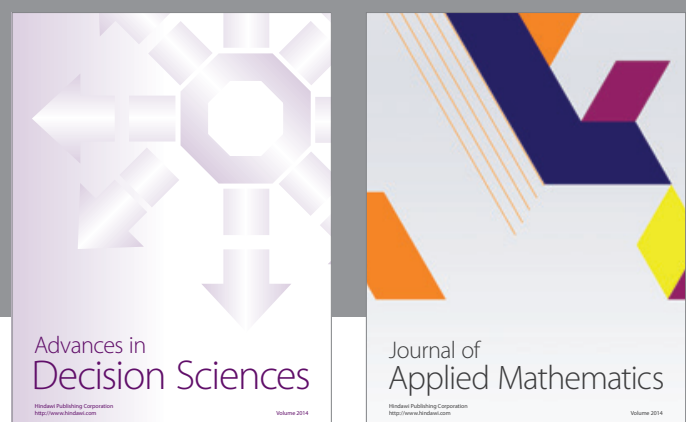

Journal of

Applied Mathematics
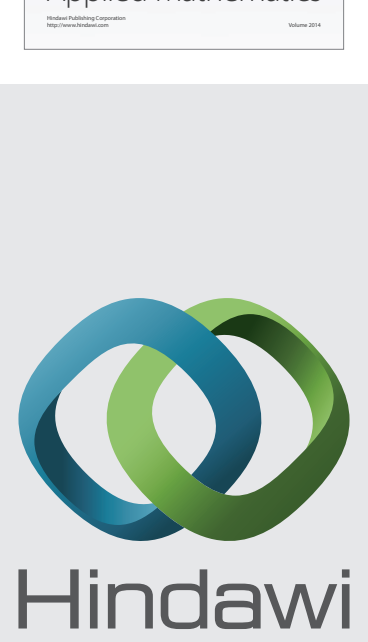

Submit your manuscripts at http://www.hindawi.com
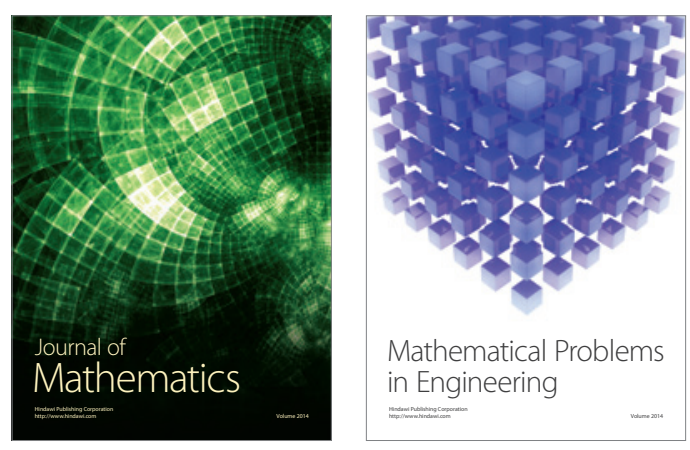

Mathematical Problems in Engineering
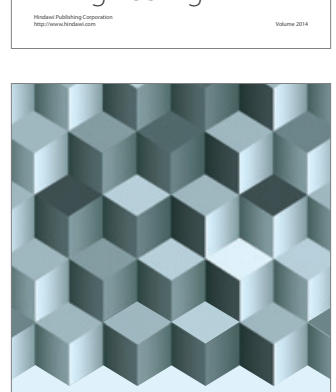

Journal of

Function Spaces
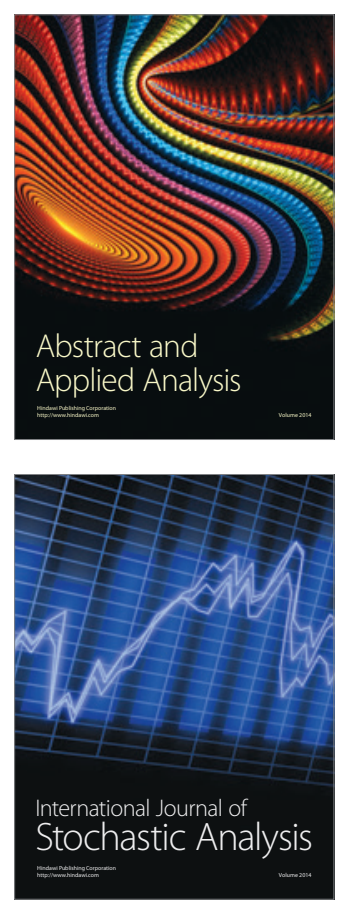

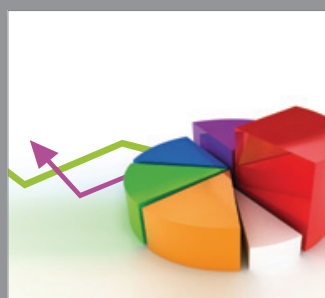

ournal of

Probability and Statistics

Promensencen
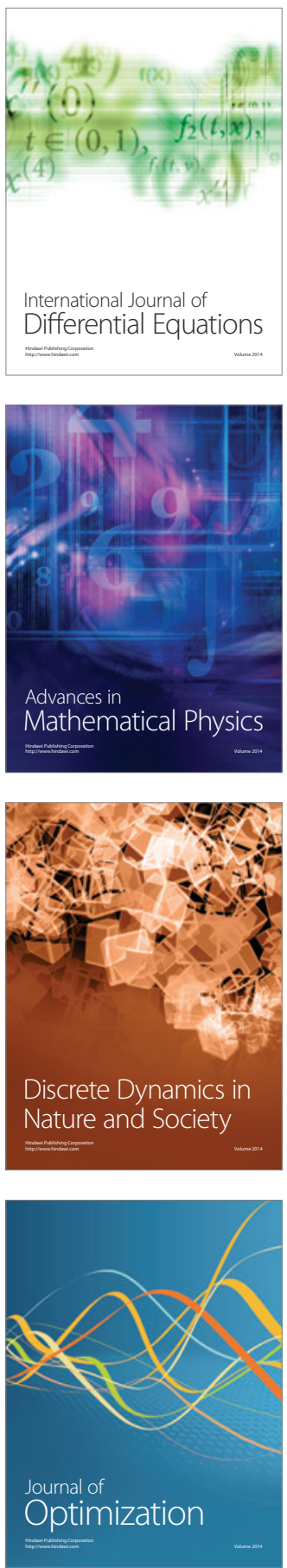